\title{
Validating Ocean General Circulation Models via Lagrangian particle simulation and data from drifting buoys
}

\author{
Karan Bedi ${ }^{10000-0001-9030-0047]}$, David Gómez-Ullate ${ }^{2,3[0000-0002-6890-6584]}$, \\ Alfredo Izquierdo ${ }^{40000-0003-3842-1460]}$, and Tomás Fernández \\ Montblanc ${ }^{5[0000-0001-6910-448 X]}$ \\ 1 Department of Mathematics, Indian Institute of Technology Roorkee, India. \\ 2 Higher School of Engineering, University of Cádiz, Puerto Real, Spain. \\ 3 Departamento de Física Teórica, Universidad Complutense de Madrid, Spain. \\ 4 Applied Physics Department, University of Cádiz, Puerto Real, 11510, Spain \\ ${ }^{5}$ Faculty of Marine Science (CACYTMAR), University of Cádiz, Puerto Real, Spain.
}

\begin{abstract}
Drifting Fish Aggregating Devices (dFADs) are small drifting platforms with an attached solar powered buoy that report their position with daily frequency via GPS. We use data of 9,440 drifting objects provided by a buoys manufacturing company, to test the predictions of surface current velocity provided by two of the main models: the NEMO model used by Copernicus Marine Environment Monitoring Service (CMEMS) and the HYCOM model used by the Global Ocean Forecast System (GOFS).
\end{abstract}

Keywords: Copernicus CMEMS · HYCOM model · Lagrangian trajectory simulation · Fish Aggregating Devices

\section{Introduction}

Drifting Fish Aggregating Devices (dFADs) are man-made objects that consist of a bamboo raft covered by old pieces of purse seine net. Throughout the 2000s, several technological improvements were added to dFADs, including the use of GPS buoys to more accurately locate dFADs and logs, and the introduction of echosounder buoys to monitor the amount of biomass aggregated under them [1]. While the reasons why the fish choose to aggregate below these devices are still under debate [2], there is no doubt that they indeed perform their task, as evidenced by the large scale deployment of dFADs on the ocean, with thousands of new buoys deployed every year.

The main purpose of these devices is naturally to help fishing companies in their task. The buoys can be equipped with other sensors and technologies that allow them to provide useful data for other scientific purposes as a by product. Data collected from dFADs have given rise to a considerable number of studies, mostly concentrating on the ecological aspects of fisheries management, and also on the echosounder technology and the reliability of their reported measures. For a good review on the subject, see [3] and the refefences therein.

ICCS Camera Ready Version 2019

To cite this paper please use the final published version:

DOI $10.1007 / 978-3-030-22747-0 \_20$ 
The purpose of this paper is to highlight the relevance of data gathered from dFADs for other scientific studies. Buoys equipped with an accelerometer can be useful to measure wave motion, their echosounder measures concentration of fish on a certain volume below them, and sensors for salinity, temperature, etc. can provide useful data to feed and validate forecasting models.

In this paper we will only use the daily position of the buoys, registered through a daily GPS report, to draw some information about the ocean currents at those points. In particular, since we have data from ca. 10,000 buoys and each of them has an average lifetime of ca.100 days, we have ca. $1 \mathrm{M}$ daily displacements, which we will test against the prediction of daily displacements obtained by Lagrangian integration of virtual particles propagating with the velocity fields provided by two OGCM models: the NEMO model used by Copernicus Marine Environment Monitoring Service (CMEMS) and the HYCOM model used by the Global Ocean Forecast System (GOFS).

\subsection{Ocean General Circulation Models (OGCMs)}

For the purpose of this study, we have used the global ocean analyses from two state-of-the-art global ocean monitoring and forecasting systems: the Global Ocean Forecast System (GOFS) and Copernicus Marine Environment Monitoring Service (CMEMS).

GOFS 3.1 analysis is a product developed by the Naval Research Laboratory (NRL), with global coverage. It uses the HYCOM model [4] with 41 vertical levels and uniform $1 / 12^{\circ}$ resolution between $80.48^{\circ} \mathrm{S}$ and $80.4^{\circ} \mathrm{N}$. It runs daily assimilating observed data through NCODA (Navy Coupled Ocean Data Assimilation [5]) system. The ocean analysis expands from July 2014 until present, with the 3D fields available every 3 hours.

GLOBAL_ANALYSIS_FORECAST_PHY_001_024 is developed by CMEMS, providing daily mean ocean physical fields with a global coverage at $1 / 12^{\circ}$ resolution. It uses version 3.1 of NEMO ocean model [6] with 50 vertical levels. It runs daily assimilating altimetry data, vertical profiles of salinity and temperature and satellite sea surface temperature (SST). CMEMS global ocean analysis expands from January 2016 until present.

Both of the models cover the region and time span for which we have data of real displacements, allowing us to compare the predictions based on different

analyses. For simplicity, in the rest of this paper we will refer to the models as HYCOM and Copernicus.

\subsection{Drifting Fish Aggregating Devices (FADs)}

Drifting Fish Aggregating Devices (dFADs) are synthetic floating devices specially designed to attract ocean going pelagic fish such as tuna, marlin, etc. They exploit the fact that many fish species naturally assemble near floating objects. dFADs usually consist of a floating raft, synthetic netting submerged in 
water, and a smart buoy equipped with echo sounder and GPS systems. dFADs carry a heavy suspended weight in order to provide stability. They estimate the fish density beneath it and transmit this information along with its position to satellites.

\subsection{Lagrangian particle simulation}

The validation of OGCMs by comparison with drifting buoys was conducted using a Lagrangian model, allowing us to calculate the predicted trajectories from the velocity field provided by the OGCM. The Lagrangian ocean analysis model Parcels v2.0.0beta was used to simulate the trajectories of the dFADs. Parcels is an open source Python based code that simulates the advection and dispersion of passive particles given a 3D hydrodynamic velocity field. The code enables a potential development in order to account for flow-particle interactions and/or more complex particle behaviour (see further details and model description in $[7,8])$. For a recent review of Lagrangian Ocean modelling, the reader is advised to see [9] and the references therein.

A careful treatment of the physical properties of drifting dFADs needs to be performed. The devices have a suspended weight to stabilize them, that usually lies 40-60 $\mathrm{m}$ below the surface, so it is unclear how to model their drifting motion: what should be their drag coefficient, at which depth are they being driven, etc. This will be the object of further study. For the preliminary analysis reported in this paper, we chose to model dFADs as virtual particles (which are just driven by the current without interaction) and we have selected current velocity fields corresponding to the surface layer. Numerical tests performed at different depths suggest that surface layer is the best choice to describe their driving force.

\section{Simulations \& Analysis}

\subsection{Exploratory Data Analysis}

Describing the Dataset Our dataset includes the daily positions of 9,440 buoys, corresponding to the period ranging from Jan 012016 to Nov 302016 . It consists of 925,187 entries, each entry having a Buoy Id (a unique identifier), Latitude-Longitude (co-ordinates for the position of the Buoy) and a timestamp (date-time when the position was recorded).

Data cleaning After eliminating entries with missing data, upon closer study it becomes evident that some of the daily displacements cannot possibly be due to drifting buoys, as these displacements are larger than $200 \mathrm{~km}$ in a single day. These anomalous displacements are likely due to the fact that the buoy was being carried by a fishing boat. A filter is applied to eliminate from the study any displacement greater than $120 \mathrm{~km}$ in a single day. The resulting trajectories are broken into smaller trajectories if such displacements occur in the middle of the original trajectory (see Figure 2). Only the new trajectories having 5 or more 
data-points after filtering are considered for further analysis. This procedure increases the number of trajectories from 9,440 to 29,241 , while it decreases the total number of observations from 925,187 to 810,165 .

This filtering process to discard non-drifting buoys is a first approach that probably needs to be refined: while all discarded displacements are probably correct, there remains the chance that some buoys carried by boats are still present in the analysis. A finer analysis will be performed, either by considering the whole time series for fixing the criterion, or by considering external information (e.g. echo sound signal) provided by the company.

A histogram with the number of observations for each buoy before and after the filtering process can be seen in Figure 2, while one trajectory with anomalous jumps has been shown for illustrative purposes in Figure 1.

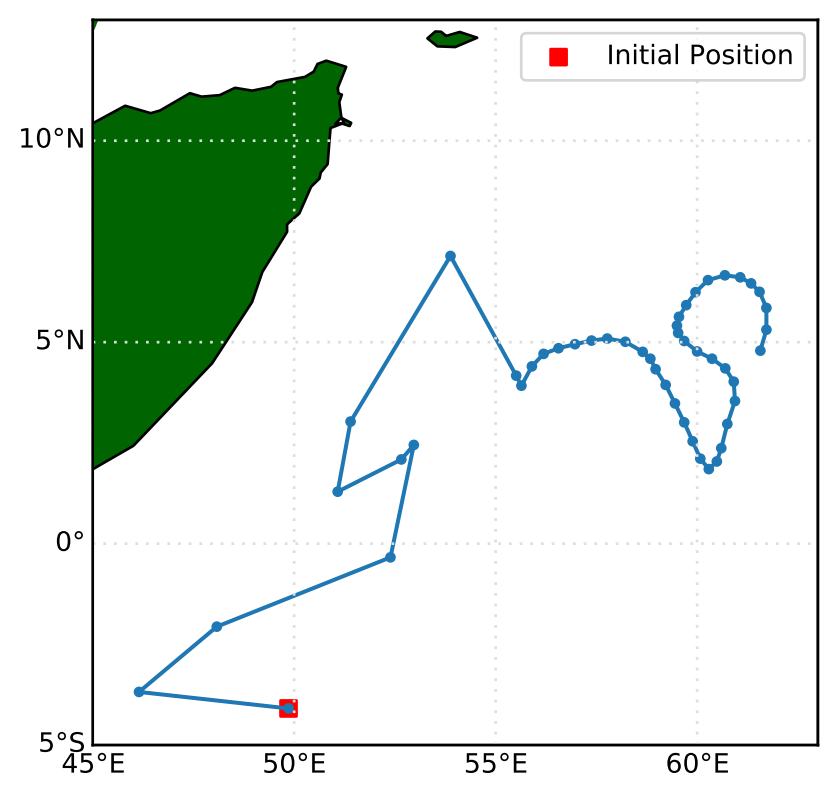

Fig. 1. Trajectory fo 49 days of a buoy with anomalous daily displacements, probably moved by external means during the first 9 days, and drifting for the rest of the days.

Data Exploration The initial position of the buoys are spread across both the Atlantic and Indian Ocean. The initial positions of the original 9,440 can be seen in Figure 4, while the initial positions of the 29,241 trajectories obtained 


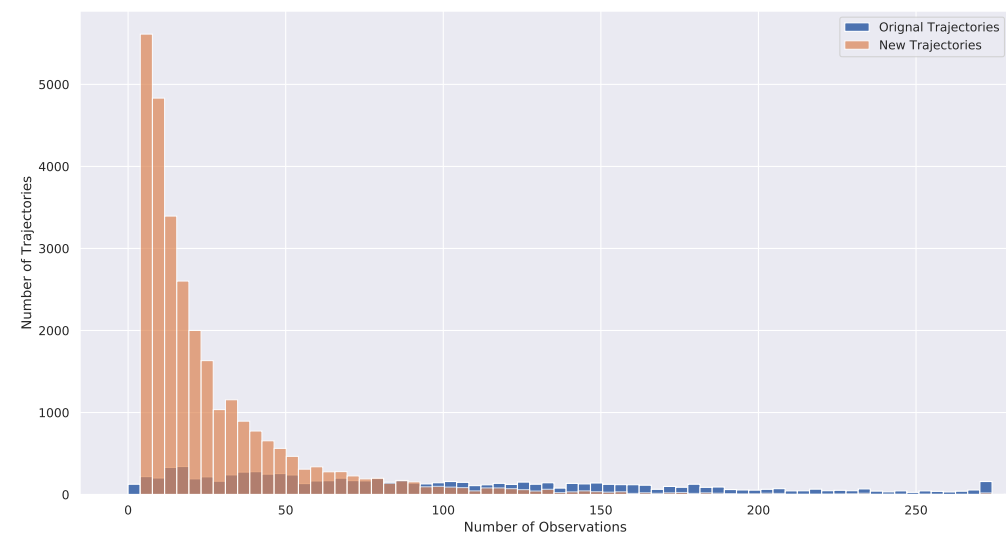

Fig. 2. Histogram of number of observations for new trajectories

after the filtering process explained above, are depicted in Figure 5. Notice in Figure 4 that there is a gap with very few initial positions of buoys off the coast of Somalia, which is probably due to security reasons, in order to avoid pirate ships. The geographical distribution of the 810,165 daily displacements can be

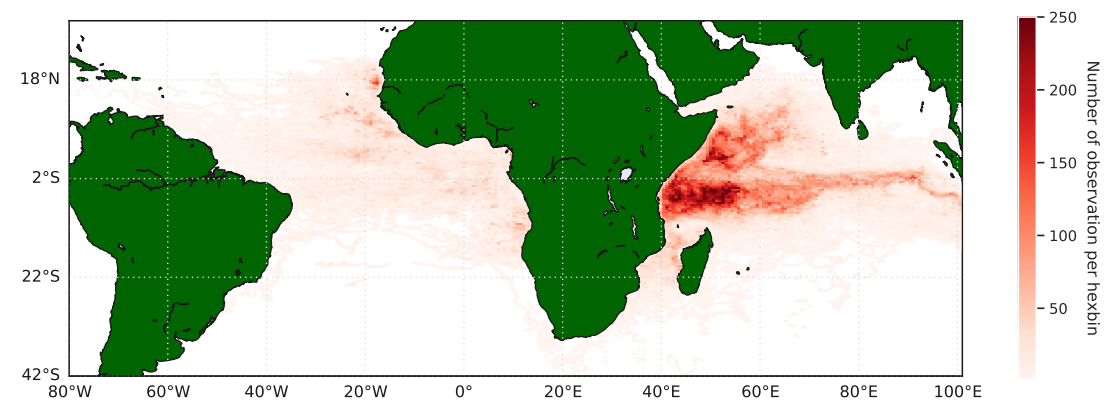

Fig. 3. Geographical distribution of the 810,165 data-points (daily positions) in the dataset

seen in Figure 3. The plot corresponds to $180^{\circ}$ longitude degrees and has been divided in 300 hexagonal units, so each unit spans an area of roughly $0.6^{\circ}$, i.e. a circle of radius approximately $30 \mathrm{~km}$. The plot shows clearly that the region around the equator in the Indian ocean has a higher concentration of data points.

ICCS Camera Ready Version 2019

To cite this paper please use the final published version:

DOI $10.1007 / 978-3-030-22747-0 \_20$ 


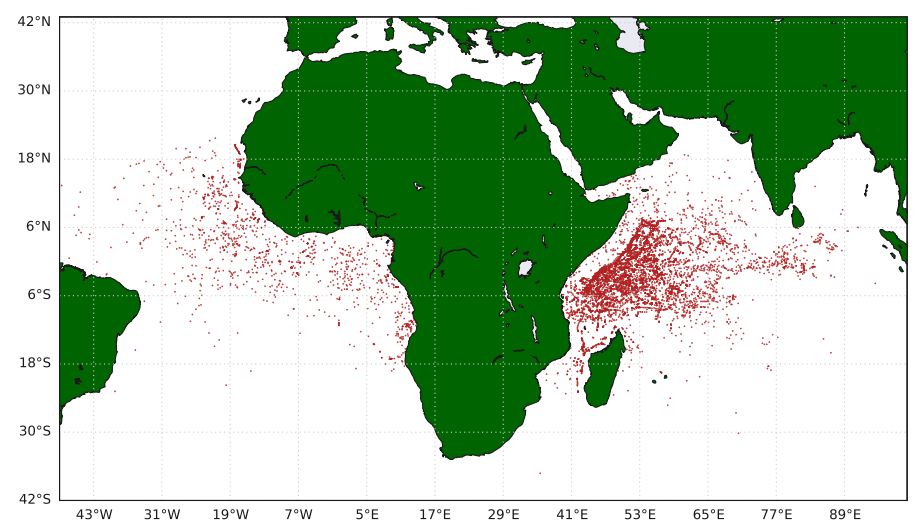

Fig. 4. Initial positions of the 9,440 original trajectories

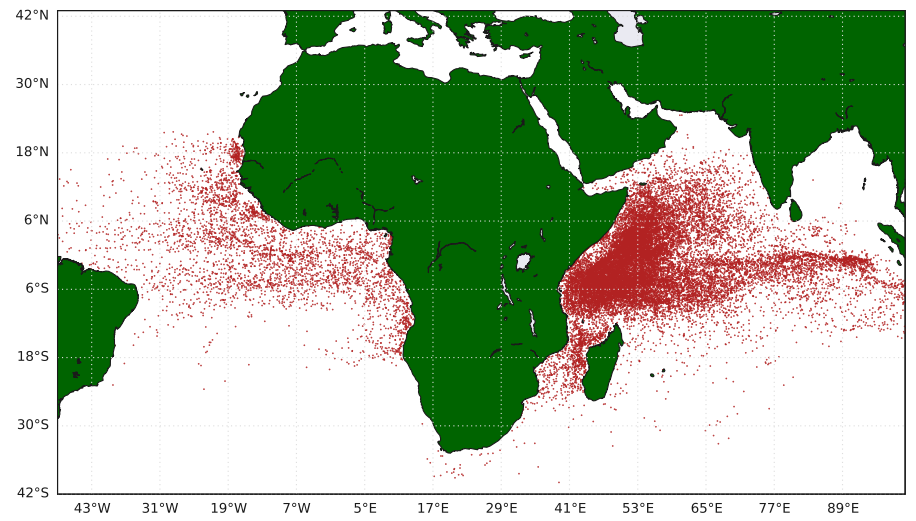

Fig. 5. Initial positions of the 29,241 new trajectories, after filtering our anomalous daily displacements

ICCS Camera Ready Version 2019

To cite this paper please use the final published version: 


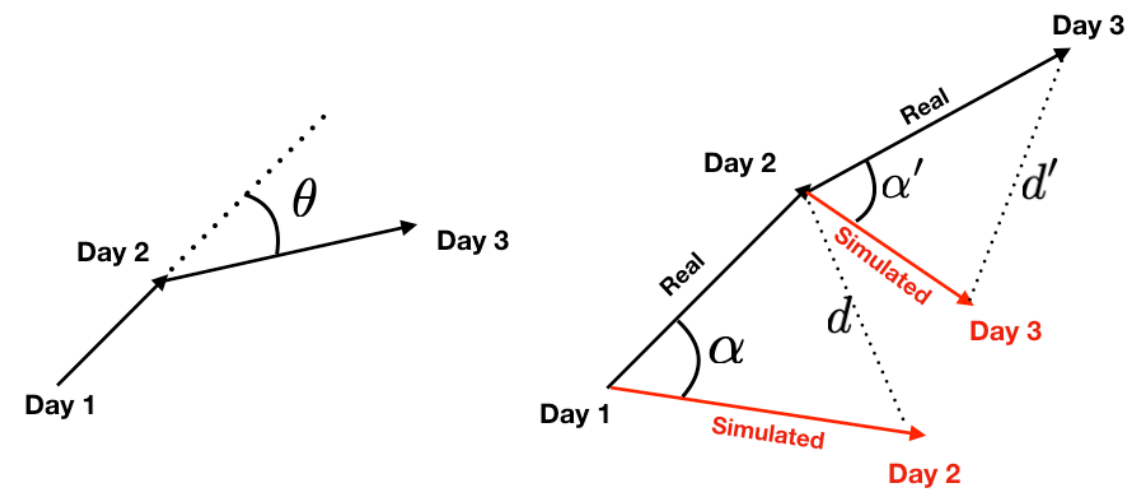

Fig. 6. Left: Angle $\theta$ between two consecutive daily displacements. Right: Angle $\alpha$ and distance $d$ between the real and simulated daily displacements.

As part of the exploratory analysis, we have studied the distribution of daily displacements and daily changes in direction from the real dataset. The daily displacements of the buoys have a mean value of $28.63 \mathrm{~km}$ (which corresponds to a mean current speed of $0.32 \mathrm{~m} / \mathrm{s}$ ) with a standard deviation of $20.88 \mathrm{~km}$, and the complete distribution can be seen in Figure 7. For convenience, we have plotted alongside the distribution of daily real displacements, also the daily displacements predicted by Lagrangian particle simulation with velocity fields taken from the HYCOM and Copernicus models. Their geographical distribution is shown in Figure 8, where we show at each cell the average of the daily displacements with origin in that cell (see Figure 3). It is clear that larger daily displacements concentrate on the areas corresponding to the most intense current systems, namely the Western Boundary Currents and the Equatorial Current systems, where mean kinetic energy values are larger. Local maxima appear corresponding to the North Brazil Current in the tropical Atlantic, at the Agulhas Current around the southern tip of Africa and at the Somalia Current, Madagascar Current system and Equatorial Current system in the Indian Ocean.

Similarly, we have studied the daily change in direction, i.e. the angle $\theta$ between two consecutive daily displacement vectors, as shown in the left panel of Figure 6 . The distribution of such angles is clearly centered around zero (has mean value

ICCS Camera Ready Version 2019

To cite this paper please use the final published version:

DOI $10.1007 / 978-3-030-22747-0 \_20$ 


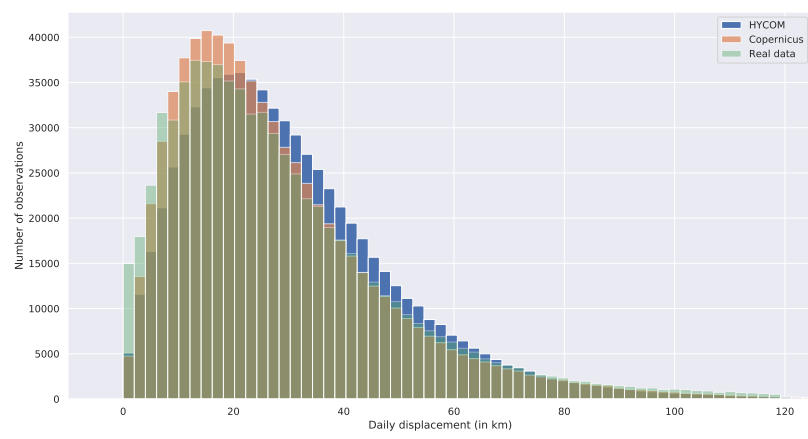

Fig. 7. Histogram of real and simulated daily displacements

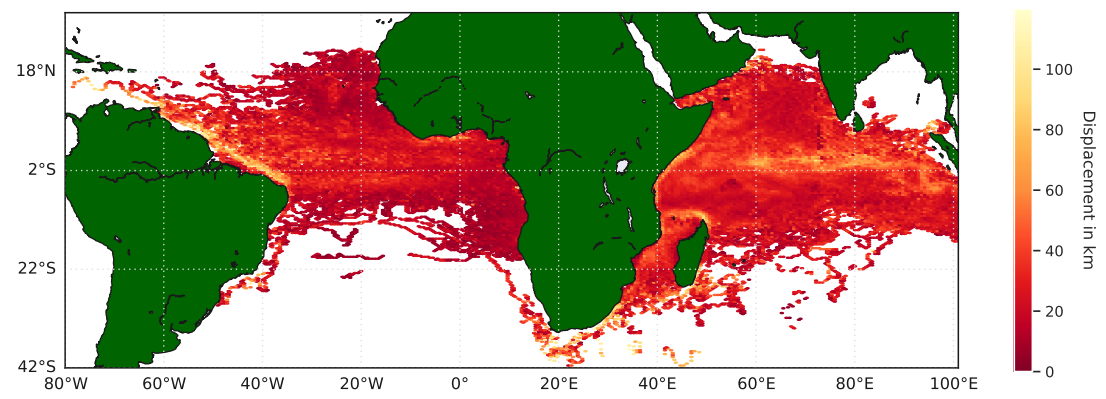

Fig. 8. Geographical distribution of daily displacements

ICCS Camera Ready Version 2019

To cite this paper please use the final published version:

DOI $10.1007 / 978-3-030-22747-0 \_20$ 
$-0.8^{\circ}$ ) with standard deviation $42^{\circ}$. Here, the geographical distribution of such angles is also quite informative (see Figure 10). We see a higher concentration of negative angle shifts (clockwise rotation) above the equator, and positive angle shifts (counter-clockwise rotation) below the equator, due to the Coriolis effect.

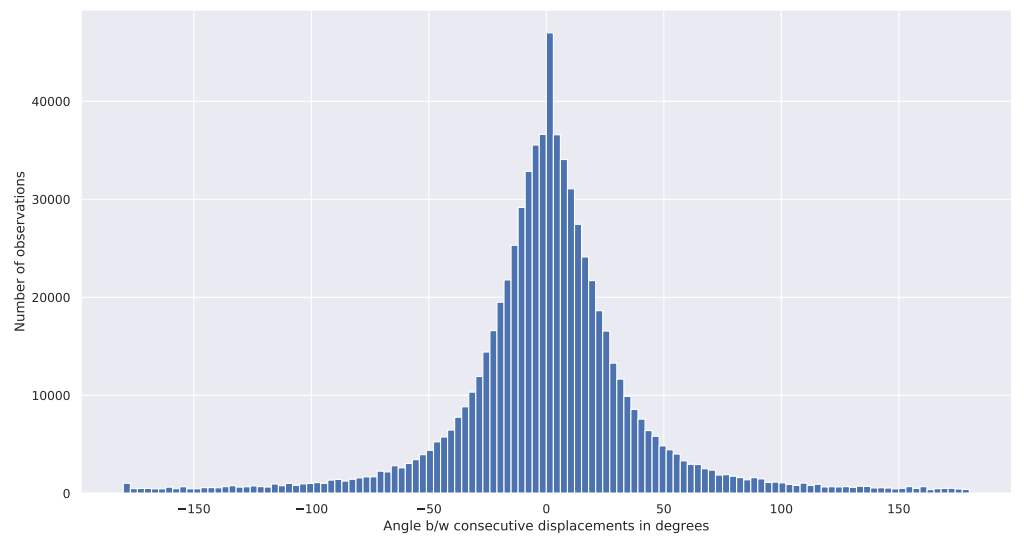

Fig. 9. Histogram of angle $\theta$ between two consecutive daily displacements in degrees

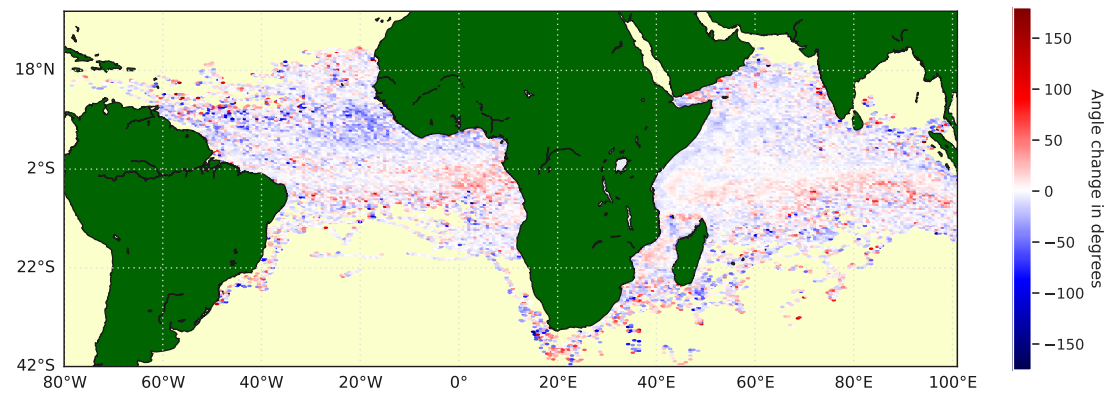

Fig. 10. Geographical distribution of angle $\theta$ between consecutive daily displacements

ICCS Camera Ready Version 2019

To cite this paper please use the final published version:

DOI $10.1007 / 978-3-030-22747-0 \_20$ 


\subsection{Simulation}

In order to evaluate the OGCMs performance, the Lagrangian model simulations were performed considering the dFADs as passive particles, i.e., their displacements are dictated purely by the ocean's velocity field and their presence does not affect the field itself. The Lagrangian model runs were conducted accounting only for the advection using as input the horizontal velocity fields at the ocean's surface provided by GOFS3.1 and CMEMS analyses and using a fourth-order Runge-Kutta integration. Other processes affecting the floating object motion in the ocean, such as flow-object interaction, wind drift, turbulence and wave motion are neglected in the model runs. Particle trajectories were calculated using a time step of 300 seconds in order to avoid particle displacements larger than OGCMs spatial resolution. The dFADs trajectories were simulated using composite of successive 24 hour runs. After each 24 hour run, the simulated position was compared to the dFADs data real position, model skill metrics were calculated, the particle position was set to the real position and the next 24 hour period was simulated again. In this manner, we have 810,165 predictions for simulated daily displacements, to be tested against the real measured displacements coming from the GPS positions of the dFADs. As mentioned in the Introduction,

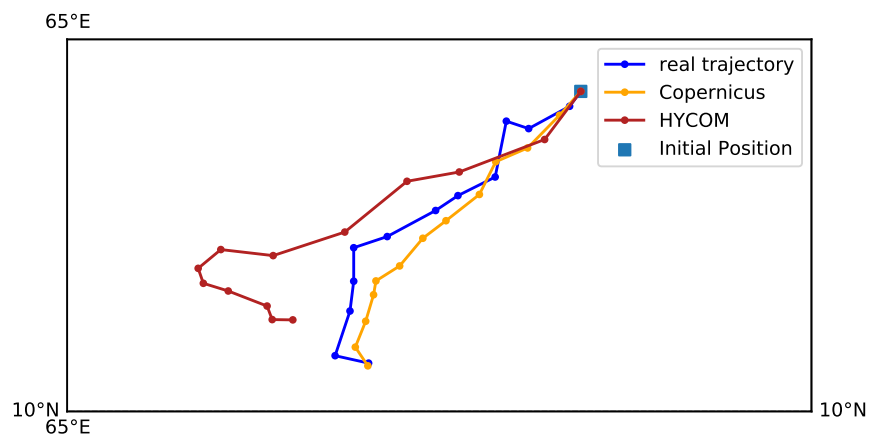

Fig. 11. Real and simulated trajectories of a Buoy for 14 days

ICCS Camera Ready Version 2019

To cite this paper please use the final published version:

DOI $10.1007 / 978-3-030-22747-0 \_20$ 
a more thorough modelling approach is needed to study the physics of the transport process: treating the dFADs as virtual particles and propagating them with the surface current being just one possible choice to serve as baseline for this model. A more elaborate understanding of the physical properties, together with an optimal choice of external parameters will probably improve the predictive results.

A cherry-picked example of real and simulated trajectories can be seen in Figure 11. It is important to stress that we are hindcasting the simulation, i.e. the velocity fields for each model have been taken from the corresponding database as the prediction for each time snapshot. Thus we compute the trajectories that the virtual particles would have taken according to HYCOM and Copernicus predictions at each time, and compare them with the real trajectories that actually took place. There are no forecasting predictions of trajectories or velocity fields in this analysis.

\section{Results}

We compare the predicted and real displacements with two metrics (see left panel of Figure 6):

1. the distance $d$ between the predicted and real final positions at the end of each day,

2. the angle $\alpha$ between the real and simulated daily prediction vectors.

After performing the Lagrangian simulation, we compute these magnitudes for each of the 810,165 points in our dataset. The results can be seen in Figure 12 for the distance $d$ and Figure 13 for the angle $\alpha$, while the main results that show the mean and standard deviations of both distributions are summarized in Table 1.

Table 1. Mean and standard deviations of the distributions depicted in Fig. 12 and Fig. 13

\begin{tabular}{|l|cc|cc|}
\hline & \multicolumn{2}{|c|}{$\alpha$} & \multicolumn{2}{c|}{$d$} \\
\hline & mean & std & mean & std \\
\hline HYCOM & $0.4^{\circ}$ & $66^{\circ}$ & 22 & 14 \\
Copernicus & $-0.5^{\circ}$ & $\mathbf{5 8}^{\circ}$ & $\mathbf{1 7}$ & $\mathbf{1 2}$ \\
\hline
\end{tabular}

\section{Conclusion}

We see from the previous analysis that, all other things being equal, the daily displacement predictions based on the NEMO model by Copernicus CMEMS are 


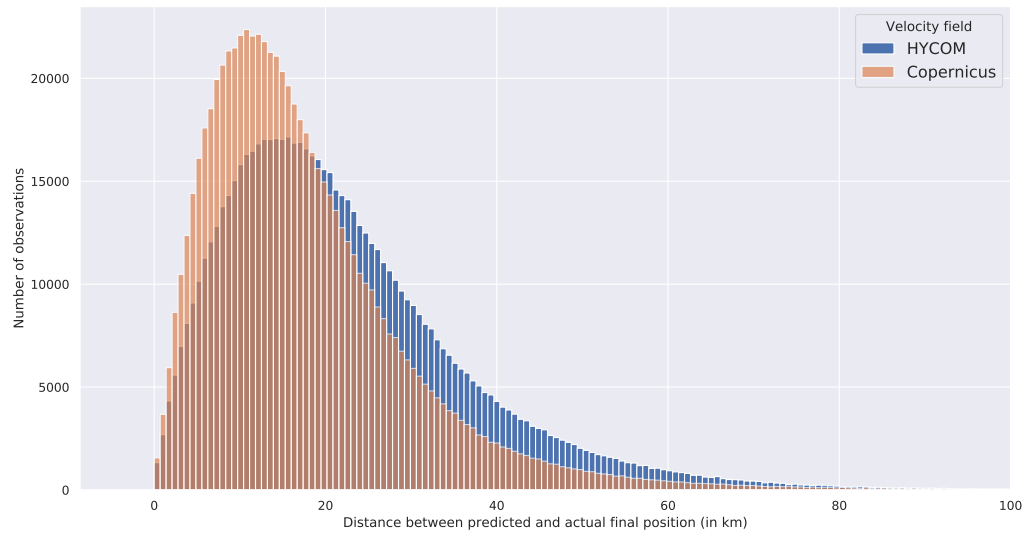

Fig. 12. Histogram of distance between predicted and actual final positions

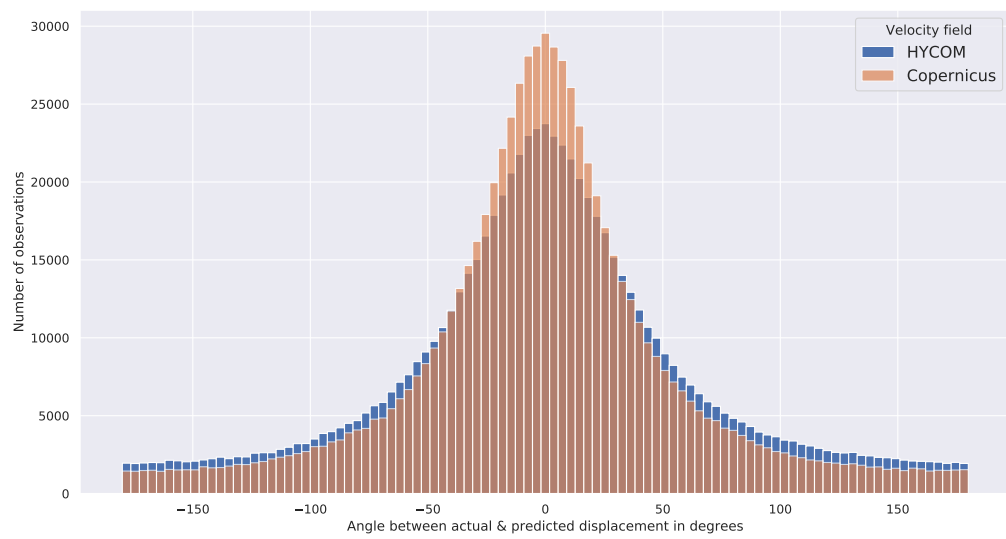

Fig. 13. Histogram of angle change in degrees

ICCS Camera Ready Version 2019

To cite this paper please use the final published version:

DOI $10.1007 / 978-3-030-22747-0 \_20$ 
slightly better (roughly $20 \%$ better) than the predictions obtained by simulating trajectories with the velocity fields predicted by the HYCOM model used by GOFS.

Large scale properties show a reasonable agreement between real and simulated data, such as the overall displacement distribution (Figure 7). The surface circulation patterns of the tropical indian and atlantic ocean are also properly reflected in the dFAD data.

This being said, it is perhaps noteworthy that the predicted direction after a day of drifting motion on the ocean misses the real direction on average by $60^{\circ}$, which seems a rather high figure considering that we are only hindcasting rather than forecasting. Likewise, and related with this, the expected distance between the predicted and real positions after one day of drifting in the ocean is (in the best case) $17 \mathrm{~km}$, almost half the expected daily displacement.

A very important remark, as a disclaimer, is that all results reported here are not a direct measure of both OGCM models, but rather an indirect measure via a specific choice of Lagrangian integration model. As it has been stressed, further study is needed to establish the best simulation model, most probably taking into account drag effects, interaction with the fluid, other numerical integration schemes, etc. However, perhaps the lesson to be learnt is that one should be very cautious about interpreting the uncertainty involved in the predictions made by these models, even if they are nowcasting. We would like to highlight also the importance of running this type of independent tests, and the relevance of dFAD data as a valuable source of information for scientific research. Given the enormous amunt of dFADs, the possibilities of incorporating additional sensors, and the near-real time communication protocols there is a huge potential for their use in monitoring the tropical oceans.

\section{References}

1. Lopez, J., Moreno, G., Boyra, G., \& Dagorn, L. (2016). A model based on data from echosounder buoys to estimate biomass of fish species associated with fish aggregating devices. Fishery Bulletin, 114(2), 166-178.

2. Castro, J., J. Santiago, and A. Santana-Ortega. 2002. A general theory on fish aggregation to floating objects: An alternative to the meeting point hypothesis. Rev. Fish Biol. 11: 255277.

3. Dempster, T., \& Taquet, M. (2004). Fish aggregation device (FAD) research: gaps in current knowledge and future directions for ecological studies. Reviews in Fish Biology and Fisheries, 14(1), 21-42.

4. Chassignet, E.P., H.E. Hurlburt, E.J. Metzger, O.M. Smedstad, J.A. Cummings, G.R. Halliwell, R. Bleck, R. Baraille, A.J. Wallcraft and C. Lozano, 2009: US GODAE Global Ocean Prediction with the HYbrid Coordinate Ocean Model (HYCOM). Oceanography vol 22, 64-75.

5. Cummings, J.A., 2005: Operational multivariate ocean data assimilation. Quart. J. Royal Met. Soc., Vol. 131, pp 3583- 3604.

6. Madec, G. and the NEMO team, 2008: NEMO ocean engine, Note du Ple de modlisation 27, Institut Pierre-Simon Laplace (IPSL),France, ISSN 12881619.

ICCS Camera Ready Version 2019

To cite this paper please use the final published version:

$\begin{array}{lll}\text { DOI } & 10.1007 / 978-3-030-22747-0 \_20\end{array}$ 
7. Lange, M. \& Sebille, E. Van. Parcels v0.9: Prototyping a Lagrangian ocean analysis framework for the petascale age. Geosci. Model Dev. (2017). https://doi.org/10.5194/gmd-10-4175-2017

8. Delandmeter, P. \& Van Sebille, E. The Parcels v2.0 Lagrangian framework: new field interpolation schemes. Geosci. Model Dev. Discuss. (2019). https://doi.org/10.5194/gmd-2018-339

9. Van Sebille,E. et al. (2018). Lagrangian ocean analysis: Fundamentals and practices. Ocean Modelling, 121, 49-75.

10. Fonteneau, A., Chassot, E., \& Bodin, N. (2013). Global spatio-temporal patterns in tropical tuna purse seine fisheries on drifting fish aggregating devices (DFADs): Taking a historical perspective to inform current challenges. Aquatic Living Resources, 26(1), 37-48.

11. Trygonis, V., Georgakarakos, S., Dagorn, L., \& Brehmer, P. (2016). Spatiotemporal distribution of fish schools around drifting fish aggregating devices. Fisheries research, 177, 39-49.

12. Taquet, M., Sancho, G., Dagorn, L., Gaertner, J. C., Itano, D., Aumeeruddy, R., ... \& Peignon, C. (2007). Characterizing fish communities associated with drifting fish aggregating devices (FADs) in the Western Indian Ocean using underwater visual surveys. Aquatic Living Resources, 20(4), 331-341.

13. Moreno, G., Dagorn, L., Capello, M., Lopez, J., Filmalter, J., Forget, F., ... \& Holland, K. (2016). Fish aggregating devices (FADs) as scientific platforms. Fisheries Research, 178, 122-129.

ICCS Camera Ready Version 2019

To cite this paper please use the final published version:

DOI $10.1007 / 978-3-030-22747-0 \_20$ 